Unidad de Litiasis. Servicio de Urología. Hospital Clínico Universidad de Chile $(\mathrm{HCUCH})$. Santiago, Chile.

Recibido el 16 de agosto de 2015, aceptado el 1 de abril de 2016.

Correspondencia a: Dr. Enrique Ossandón Salas Santos Dumont 999, Independencia, Santiago, Chile. enriqueossandon@mi.cl

\section{Impacto de la profilaxis secundaria en el manejo de pacientes con urolitiasis de alto riesgo de recidiva}

\author{
ENRIQUE OSSANDÓN S., FRANCISCO SEPÚLVEDA T., \\ CRISTIÁN ACEVEDO C.
}

\section{Impact of secondary prophylaxis in the management of patients with high recurrence risk of urolithiasis}

Background: In a previous study, we showed our experience in a group of 54 patients with a high risk of urolithiasis recurrence, who were subjected to a complete metabolic evaluation. Aim: To report the evolution of these patients after 5 years of follow-up. Patients and Methods: All patients underwent a general management of urolithiasis plus specific treatments for underlying metabolic disorders. Each patient had an annual medical assessment including a clinical examination, urinalysis and imaging studies (non-enhanced computed tomography scan, ultrasonography and plain abdominal $R x$ rays). In every case, the underlying metabolic disorder, treatment adherence, stones on imaging studies and symptomatology were evaluated. Adherence of general and specific measures were evaluated subjectively. Failure of secondary prevention was defined as the recurrence of clinical or imaging urolithiasis (increase of the number of lithiasis) despite a correct treatment of the metabolic disorders. Results: Twenty nine patients completed the follow-up. Mean age was 45 years old. Nineteen patients (65\%) had only one metabolic disorder, three patients (10\%) two disorders, one patient (3\%) four disorders, and six patients (21\%) a normal metabolic study. The median of follow-up was 54 months (45-60). During that period, twenty-three patients (79\%) kept the treatment as it was indicated. In this subgroup, 21 had no clinical or imaging recurrence of urolithiasis during follow-up (91\%). Total adherence to treatment and follow-up was 42\% (23/54) of the initial group of patients. Conclusions: A complete metabolic study allows to identify patients with a high risk of urolithiasis recurrence, enabling a specific treatment of the metabolic disorder. Our experience shows that 75\% (21/29) of patients remain free of recurrence at five years of follow-up.

(Rev Med Chile 2016; 144: 710-715)

Key words: Recurrence; Secondary Prevention; Urolithiasis.
L a litiasis de vía urinaria es una patología de alta prevalencia, alcanzando hasta en $15 \%$ de la población y puede variar de gran manera de acuerdo al lugar geográfico estudiado ${ }^{1,2}$. Se han descrito diversos factores asociados a un mayor riesgo de presentar esta patología, entre los que destacan una baja ingesta de líquidos, y una elevada ingesta de proteínas de origen animal y sodio ${ }^{3}$. El riesgo de recurrencia después de un episodio de urolitiasis puede llegar a $45 \%$ a 5 años y $75 \%$ a 20 años $^{4}$, porcentaje no menor, considerando la morbilidad y costos que puede significar 
un nuevo episodio (cólico renal, urosepsis, falla renal). Numerosos trabajos han demostrado que hasta $80 \%$ de los pacientes presenta una o más alteraciones metabólicas como factor de riesgo de formación de litiasis ${ }^{5,6}$. Con el objetivo de disminuir la recurrencia de episodios de litiasis, es fundamental realizar una profilaxis secundaria dirigida específicamente a la alteración metabólica subyacente, asociado a medidas generales. Dentro de las medidas generales más usadas para reducir la recurrencia de cálculos se encuentra un aumento de la ingesta de líquido que facilite una diuresis mayor de dos litros diaria. Esta única medida lograría reducir las recurrencias en hasta $40 \% 7$. Otras medidas generales, como la dieta hipoproteica, hiposódica y moderada en calcio, permitirían reducir en $50 \%$ los eventos litiásicos en comparación con el grupo que sólo reduce el calcio de la dieta ${ }^{8}$. En cambio, las medidas específicas comprenden el uso de medicamentos como alopurinol, hidroclorotiazida y citrato de potasio, la reducción del oxalato de la dieta conservando la ingesta de calcio, y la paratiroidectomía.

Previamente comunicamos nuestra experiencia de 54 pacientes con riesgo elevado de recurrencia de urolitiasis, en los que realizamos una evaluación metabólica completa ${ }^{9}$. En nuestra experiencia, $65 \%$ de los pacientes con urolitiasis recurrente presentó una o más alteraciones metabólicas. Todos estos pacientes se sometieron a medidas profilácticas generales y aquellos con alteración metabólica recibieron, además, una profilaxis secundaria específica. El objetivo de este trabajo es evaluar la recurrencia de estos pacientes intervenidos con medidas profilácticas generales y específicas durante cinco años de seguimiento.

\section{Pacientes y Método}

Tal como describimos en nuestro trabajo previo, realizamos un estudio metabólico completo en 54 pacientes con alto riesgo de recurrencia de urolitiasis. Este selecto grupo de pacientes fue definido por criterios de recurrencia, magnitud de la patología inicial al momento del diagnóstico, o por las serias consecuencias potenciales que tendría un nuevo episodio (paciente monorreno). Los criterios de inclusión considerados para realizar un estudio metabólico y someter a una prevención secundaria fueron la litiasis recurrente en un período inferior a 5 años, nefrolitiasis múltiple uni o bilateral, litiasis coraliforme, litiasis radiolúcida, pacientes con un primer episodio antes de los 25 años de edad y urolitiasis en pacientes con riñón único.

En todos los pacientes se realizó una intervención con medidas generales, consistente en aumento de la ingesta de líquidos para lograr una diuresis mayor de $2.000 \mathrm{~mL}$ al día, disminución del consumo de sal ( $2 \mathrm{~g} /$ día $)$ y reducción de proteínas de origen animal hasta un máximo de 3 veces por semana. De acuerdo a la alteración metabólica subyacente, los pacientes fueron tratados además con hidroclorotiazida $50 \mathrm{mg} /$ día (pacientes con hipercalciuria renal o absortiva), citrato de potasio $1 \mathrm{~g} /$ día (pacientes con hipocitraturia), alopurinol $300 \mathrm{mg} /$ día (en aquellos pacientes con hiperuricemia y/o hiperuricosuria) o paratiroidectomía (hiperparatiroidismo primario). En casos de hiperoxalaturia se indicó una reducción en la ingesta de alimentos ricos en oxalato, procurando conservar una ingesta adecuada de calcio en la dieta.

Los pacientes fueron controlados anualmente mediante un examen clínico, análisis de orina y estudio imagenológico (tomografía computada no contrastada, ecografía y radiografía simple). Todos los pacientes contaron con tomografía computada al inicio y al final del seguimiento, $y$ en 5 pacientes se alternó con ecografía y/o radiografía simple por razones de costo y reducción a la exposición de radiaciones ionizantes. Adicionalmente, los pacientes fueron controlados en caso de cólico renal. En cada caso se evaluó la alteración metabólica subyacente, la adherencia/ abandono de tratamiento, la presencia de urolitiasis y la presencia de síntomas asociados. Se definió como fracaso de profilaxis secundaria la progresión imagenológica (aumento del número de cálculos) a pesar de un tratamiento adecuado de las alteraciones metabólicas o la eliminación de nuevos cálculos ausentes en controles previos. La eliminación de un cálculo ya presente al inicio del seguimiento no se considera fracaso terapéutico.

Los análisis se realizaron con estadística descriptiva a través de tablas y análisis de frecuencias. Se aplicó test exacto de Fisher para estudiar la asociación entre abandono de tratamiento y recurrencia de urolitiasis. Se usó el programa estadístico Stata 11. 


\section{Resultados}

Veintinueve pacientes de los 54 inicialmente estudiados completaron el seguimiento, que corresponde a $53,7 \%$ del total de pacientes inicialmente estudiados. Las características de los 29 pacientes se describen en la Tabla 1. Diecinueve pacientes $(65 \%)$ eran hombres. La edad promedio del grupo fue de 45 años (22-76). Las alteraciones metabólicas de los pacientes incluidos se describen en la Tabla 2. Se destaca que 6 pacientes $(20,7 \%)$ no tenían alteraciones en el estudio metabólico. Diecinueve pacientes $(65,5 \%)$ presentaron una única alteración metabólica, 3 pacientes $(10,3 \%)$ dos alteraciones, y un paciente $(3,4 \%)$ presentó 4 alteraciones metabólicas.

En relación a la profilaxis secundaria, a todos los pacientes se les indicó medidas generales, además del manejo específico en aquellos casos con alteración metabólica detectada. Veintitrés pacientes se mantuvieron en el manejo específico de sus alteraciones metabólicas descritas en la Tabla 2. Dos pacientes con hiperparatiroidismo primario fueron sometidos a paratiroidectomía. De acuerdo a la alteración metabólica subyacente, 5 pacientes fueron tratados con alopurinol, 5 con citrato de potasio, 5 con hidroclorotiazida, 1 con alopurinol + citrato de potasio, 1 con alopurinol + hidroclorotiazida y 4 pacientes se mantuvieron con dieta restrictiva de oxalato (Tabla 2).

El promedio de seguimiento del grupo de pacientes fue de 54 meses (45-60). Durante este
Tabla 1. Características de los pacientes

\begin{tabular}{|lcc|}
\hline Variables & $\mathbf{n}$ & \% \\
Total pacientes & 29 & 100 \\
Sexo & & \\
Hombres & 19 & 65 \\
Mujeres & 10 & 35 \\
Edad (promedio, rango) & 45 & $(22-76)$ \\
Indicación de estudio & & \\
Recurrente & 16 & 55 \\
Bilateral & 8 & 27,5 \\
Monorreno & 2 & 7 \\
Coraliforme & 2 & 7 \\
Radio-lúcida & 1 & 3,5 \\
Tratamiento primario & & \\
LEC & 19 & 65 \\
Eliminación espontánea & 5 & 17,5 \\
Nefrolitectomía percutánea & 3 & 10,5 \\
Nefrectomía & 1 & 3,5 \\
URS & 1 & 3,5 \\
\hline
\end{tabular}

LEC: litotripsia extracorpórea, URS: ureterolitectomía endoscópica.

período, 24\% de los pacientes (7/29) mostró recurrencia de episodios de litiasis. Cinco de estos pacientes presentaron aparición de nuevas litiasis en tomografía computada sin contraste, mientras que dos pacientes debutaron como cólico renal. Por el contrario, $76 \%$ de los pacientes (22/29) con intención de tratamiento se mantuvo sin progresión litiásica.

Tabla 2. Alteración metabólica y manejo específico realizado

\begin{tabular}{|c|c|c|}
\hline Alteración metabólica & n $\quad(\%)$ & Manejo \\
\hline Normal & $6(20,7)$ & General \\
\hline Hiperparatiroidismo primario & $2 \quad(6,9)$ & Paratoroidectomía \\
\hline Hiperuricosuria & $3(10,3)$ & Alopurinol 300 mg/día \\
\hline Hiperoxalaturia & $4(13,8)$ & Restricción de oxalato en dieta \\
\hline Hipercalciuria renal/absortiva & $5(17,2)$ & Hidroclorotiazida 50 mg/día \\
\hline Hipocitraturia & $5(17,2)$ & Citrato de potasio $1 \mathrm{~g} /$ día \\
\hline Hiperuricemia + Hiperuricosuria & $2 \quad(6,9)$ & Alopurinol 300 mg/día \\
\hline Hipocitraturia + Hiperuricemia & $1 \quad(3,4)$ & Alopurinol 300 mg/día + citrato de potasio 1 g/día \\
\hline $\begin{array}{l}\text { Hipercalciuria + Hiperuricemia + Hiperuricosuria + } \\
\text { Hiperoxalaturia }\end{array}$ & $1 \quad(3,4)$ & Alopurinol 300 mg/día + hidroclorotiazida 50 mg/día \\
\hline
\end{tabular}

A todos los pacientes (29) se les indicó medidas generales, más el manejo específico según la(s) alteración(es) detectadas en el estudio metabólico. 
Tabla 3. Caracterización metabólica de pacientes según adherencia al tratamiento

\begin{tabular}{|c|c|c|c|}
\hline Alteración metabólica & No abandonan & Abandonan & Total \\
\hline Hipocitraturia & 3 & 2 & 5 \\
\hline Hiperuricosuria & 2 & 1 & 3 \\
\hline Hiperoxalaturia & 3 & 1 & 4 \\
\hline Hipercalciuria & 4 & 1 & 5 \\
\hline Hiperuricosuria + Hiperuricemia & 2 & - & 2 \\
\hline Hipocitraturia + Hiperuricemia & 1 & - & 1 \\
\hline Hipercalciuria + Hiperuricemia + Hiperuricosuria + Hiperoxalaturia & 1 & - & 1 \\
\hline Hiperparatiroidismo primario & 2 & - & 2 \\
\hline Sin alteración & 5 & 1 & 6 \\
\hline Total & 23 & 6 & 29 \\
\hline
\end{tabular}

Tabla 4. Recurrencia de litiasis y abandono de tratamiento

\begin{tabular}{|lrrrrr|r|}
\hline Abandonan el tratamiento & \multicolumn{2}{c}{ Con recurrencia (\%) } & \multicolumn{2}{c|}{ Sin recurrencia (\%) } & \multicolumn{2}{c|}{ Total (\%) } \\
\hline No abandonan & 2 & $(28,6)$ & 21 & $(95,5)$ & 23 & $(79,3)$ \\
\hline Abandonan & 5 & $(71,4)$ & 1 & $(4,5)$ & 6 & $(20,7)$ \\
\hline Total & 7 & $(100,0)$ & 22 & $(100,0)$ & 29 & $(100,0)$ \\
\hline
\end{tabular}

Test exacto de Fisher, $\mathrm{p}=0,001$.

Hubo 6 pacientes (21\%) que abandonaron la profilaxis secundaria (general o específica). Cinco de estos 6 pacientes fueron los que presentaron recurrencia de urolitiasis. Las alteraciones metabólicas de estos 5 pacientes fueron: hipocitraturia en 2 pacientes, hiperuricosuria en 1 paciente, hiperoxaluria en 1 paciente e hipercalciuria en 1 paciente. Por el contrario, 23 pacientes (79\%) mantuvieron el tratamiento indicado (Tabla 3). De este grupo, 21 (91\%) pacientes se mantuvieron sin recurrencia durante el seguimiento, fracasando sólo dos pacientes (9\%). Ambos pacientes presentaban un estudio metabólico normal. Aplicando test de Fisher se observa que el grupo que abandonó la profilaxis presentó una recurrencia litiásica mayor en comparación con el grupo que mantuvo la profilaxis, con diferencia estadísticamente significativa (Tabla 4).

\section{Discusión}

En nuestra serie previa de 54 pacientes sometidos a un estudio metabólico, $65 \%$ de los pacientes presentó una o más alteraciones metabólicas ${ }^{9}$. Esto corrobora la importancia de realizar este tipo de evaluación en aquellos pacientes con un alto riesgo de recurrencia de urolitiasis y de esta manera disminuir la morbilidad y costos asociados a un nuevo episodio ${ }^{10}$. Más importante aun, en el grupo de 29 pacientes que completó el seguimiento durante 5 años, hubo 6 pacientes que abandonaron la profilaxis y de ellos 5 presentaron recurrencia de urolitiasis. Por el contrario, en 23 pacientes que mantuvieron medidas profilácticas específicas y/o generales, sólo dos pacientes recurrieron, ambos con estudios negativos para alteraciones metabólicas. Ambos resultados nos sugieren que una terapia médica específica es altamente efectiva en la prevención de nuevos episodios de urolitiasis en pacientes de alto riesgo. Asimismo, la recurrencia de los pacientes manejados con medidas generales puede corresponder a casos de subdiagnóstico de alteraciones metabólicas no detectadas.

Un problema fundamental es la adherencia a la profilaxis y seguimiento. Van Drongelen y cols. publicaron que, en un seguimiento de 5 años, sólo 
$36 \%$ de los pacientes se mantienen en tratamiento $^{11}$. En nuestro estudio, logramos un seguimiento de $54 \%(29 / 54)$ y dentro de este grupo una adherencia de $79 \%(23 / 29)$. Los pacientes refieren el abandono de la prevención tanto por los costos de la terapia o por el hecho de encontrarse libre de síntomas. En este sentido, Siener y cols. ${ }^{10}$, en una serie de 134 pacientes con múltiples episodios de urolitiasis, describe que el incremento de líquidos y el uso de alcalinizantes de la orina son las medidas que presentan una mayor adherencia por parte de los pacientes. Otros autores ${ }^{13,14}$ refieren que, a menos que los pacientes presenten un nuevo episodio de urolitiasis en los primeros meses, la mayoría de los pacientes habrá retomado su dieta previa a los 9 meses, abandonando las medidas generales de prevención.

El objetivo de nuestro trabajo es mostrar de una manera descriptiva el impacto de la profilaxis secundaria, tanto específica como general, en 29 pacientes sometidos a una evaluación metabólica por urolitiasis de alto riesgo de recurrencia. Nuestros resultados muestran que después de casi 5 años de seguimiento promedio, $76 \%$ de los pacientes con intención de tratamiento se encuentra libre de urolitiasis, y esta proporción aumenta a $91 \%$ en aquel grupo que efectivamente realizó la profilaxis general y específica. Sin embargo, debemos ser cuidadosos al momento de analizar estos resultados, ya que además de las medidas especificas para cada alteración metabólica, en todos los pacientes se indicó un incremento en la ingesta de líquidos, un bajo consumo de sal y reducción de proteínas de origen animal.

En dos estudios clínicos aleatorizados, Borghi y Curham ${ }^{8,16}$ asignaron un grupo de pacientes a una ingesta elevada de líquidos para orinar 2-2,5 litros/día versus una dieta normal. En ambos estudios se evidenció que los pacientes que incrementaban su ingesta de líquidos reducían el riesgo de tener una recurrencia de urolitiasis en $61 \%$. Por otro lado Borghi y cols. ${ }^{15}$ en otro estudio demostraron una reducción de recurrencia de urolitiasis en aquellos pacientes sometidos a una dieta baja en proteínas de origen animal y baja en sodio ( $20 \%$ vs $38 \%)$.

Los medicamentos profilácticos más usados en la actualidad son las tiazidas, el citrato de potasio y el alopurinol. Las tiazidas inducen la reabsorción de calcio y excreción de sodio y potasio en el túbulo distal, por lo tanto, generan hipocalciuria e hipokalemia. Dado que la hipokalemia induce hipocitraturia, es recomendable asociar las tiazidas a citrato de potasio ${ }^{17}$. Está demostrado que el uso de tiazidas en pacientes con hipercalciuria reducen la tasa de recurrencia litiásica ${ }^{18}$. Así también, el uso de citrato de potasio en pacientes con hipocitraturia u orinas ácidas reduciría en hasta 91\% la tasa de recurrencia litiásica ${ }^{19}$. El alopurinol inhibe la xantina oxidasa, reduciendo el ácido úrico sérico y su excreción urinaria, impidiendo la formación de pequeños núcleos de ácido úrico a los que se pueden agregar oxalato de calcio. De esta forma, el alopurinol reduciría significativamente la recurrencia de cálculos de ácido úrico y también de oxalato de calcio en pacientes con hiperuricosuria demostrada ${ }^{20}$.

Aunque estos estudios muestran que las medidas preventivas generales tienen resultados significativos en la reducción de nuevos episodios de urolitiasis, nuestro estudio sugiere que asociarla a una profilaxis específica según la alteración metabólica subyacente impactaría favorablemente en la reducción de nuevos episodios. En nuestro medio, no hay registro de estudios que evalúen el impacto de la prevención de urolitiasis con la combinación de medidas generales y específicas, ya sea con uno o más fármacos.

Dentro de las limitaciones de este trabajo se encuentran el bajo número de pacientes que completaron el seguimiento y la naturaleza descriptiva no prospectiva del mismo, en el cual nos limitamos a comparar el grupo que mantuvo la profilaxis con aquel que la abandonó. No fue posible estudiar la asociación entre las alteraciones metabólicas específicas y la recurrencia, debido al reducido número de pacientes en cada grupo.

\section{Conclusión}

Una evaluación metabólica completa permite detectar una alteración en $65 \%$ de los pacientes con un alto riesgo de recurrencia de urolitiasis $y$, de esta manera, realizar una terapia dirigida al trastorno subyacente. En nuestra experiencia, 76\% de los pacientes que inició una terapia general/ específica (intención de tratamiento) se mantuvo libre de urolitiasis después de 5 años, y se elevó a $91 \%$ en aquel grupo que mantuvo la adherencia. Es fundamental aumentar la adherencia a la terapia para mejorar aun más estos resultados. 


\section{Referencias}

1. Robertson WG, Peacock M, Baker M, Marshall DH, Pearlman B, Speed R. Studies on the prevalence and epidemiology of urinary stone disease in men in Leeds. Br J Urol 1983; 55: 595-8.

2. Andersen DA. Environmental factors in the aetiology of urolithiasis. In: Urinary Calculi; ed. Cifuentes Delatte L, Rapado A, Hodgkinson A. Pp. 130-144. 1973, Karger Publishers. Basel, Switzerland.

3. Fink H, Akornor J, Garimella P, MacDonald R, Cutting A, et al. Diet, Fluid, or Supplements for Secondary Prevention of Nephrolithiasis: A Systematic Review and Meta-Analysis of Randomized Trials. Eur Urol 2009; 56: 72-80.

4. Coe FL, Worcester EM. Calcium kidney stones. N Engl J Med 2010; 363: 954-63.

5. Robertson WG, Peacock M, Hodgkinson A. Dietary Changes and the incidence of urinary calculi in the U.K. between 1958 and 1976. J Chronic Dis 1979; 32: 469-76.

6. Andersen DA. Historical and geographical differences in the pattern of incidence of urinary stones considered in relation to possible aetiological factors. En: Proc Renal Stone. London, Churchill 1969; 7-31.

7. Curhan GC, Willett WC, Rimm EB, Stauffer MJ. A prospective study of dietary calcium and other nutrients and the risk of symptomatic kidnay stones. N Engl J Med 1993; 328: 833-8.

8. Borghi L, Schianchi T, Meschi T, Guerra A, Allegri F, Maggiore U, et al. Comparison of two diets for the prevention of recurrent stones in idiopathic hypercalciuria. N Engl J Med 2002; 346 (2): 77-84.

9. Ossandón E, Storme O, Ledesma R, Marchant F, Palma C. Resultados del estudio metabólico en 54 pacientes con urolitiasis de alto riesgo de recurrencia. Act Urol Esp 2009; 33: 429-32.
10. Sur RL, Preminger GM. Medical Treatment: Worthwhile and When? EAU Update Series 2005; 3: 10-6.

11. Van Drongelen J, Kiemeney LA, Debruyne FM, de la Rosette JJ. Impact of urometabolic evaluation on prevention of urolithiasis: a retrospective study. Urology 1998; 52: 384-91.

12. Siener R, Glatz S, Nicolay C, Hesse A. Prospective Study on the Efficacy of a Selective Treatment and Risk Factors for Relapse in Recurrent Calcium Oxalate Stone Patients. Eur Urol 2003; 44: 467-74.

13. Norman RW, Bath SS, Robertson WG, Peacock M. When should patients with symptomatic stone disease be evaluated metabolically? J Urol 1984; 132: 1137-9.

14. Robertson WG. Medical management of urinary stone disease. European Urology Update Series 1998; 7: 13944.

15. Borghi L, Meschi T, Amato F, Briganti A, Novarini A, Giannini A. Urinary volume, water and recurrences in idiopathic calcium nephrolithiasis: a 5-year randomized prospective study. J Urol 1996; 155: 839-43.

16. Curham GC, Willett WC, Rimm ED, Spiegelman D,Stauffer MJ. Prospective study of beverage and the risk of kidney stones. Am J epidemiol 1996; 143: 240-7.

17. Spernat D, Kourambos J. Urolithiasis. Medical therapies. BJU International Australia and New Zealand Supplement 2011; 108, (supplement 2): 9-13.

18. Escribano J, Balaguer A, Pagone F, Feliu A, Roque I, Figuls M. Pharmacological intervntions for preventing complication in idiopathic hypercalciuria. Cochrane Database Syst Rev 2009; 1: CD004754.

19. Fabris A, Lupo A, Bernich P, et al. Long term treatment with potassium citrate and renal stones in medullary sponge kidney. Clin J Am Soc Nephrol 2010; 5: 1663-8.

20. Ettinger B, Tang A, Citron JT, Livermore B, Williams $\mathrm{T}$, Randomized trial of allopurinol in the prevention of calcium oxalate calculi. N Engl J Med 1986; 315: 1386-9. 